\title{
Method for applying daytime colors to nighttime imagery in realtime
}

\author{
Maarten A. Hogervorst ${ }^{*}$, Alexander Toet \\ TNO Human Factors, P.O. Box 23, 3769 ZG Soesterberg, the Netherlands
}

\begin{abstract}
We present a fast and efficient method to derive and apply natural colors to nighttime imagery from multiband sensors. The color mapping is derived from the combination of a multiband image and a corresponding natural color reference image. The mapping optimizes the match between the multiband image and the reference image, and yields a nightvision image with colors similar to that of the daytime image. The mapping procedure is simple and fast. Once it has been derived the color mapping can be deployed in realtime. Different color schemes can be used tailored to the environment and the application. The expectation is that by displaying nighttime imagery in natural colors human observers will be able to interpret the imagery better and faster, thereby improving situational awareness and reducing reaction times.
\end{abstract}

Keywords: image fusion, false color, natural color, realtime fusion, lookup tables

\section{INTRODUCTION}

Night vision cameras are widely used for military and law enforcement applications related to surveillance, reconnaissance, intelligence gathering, and security. Until recently a gray- or greenscale representation of nightvision imagery has been the standard. However, the increasing availability of fused and multiband infrared and visual nightvision systems has led to a growing interest in the color display of night vision imagery ${ }^{1-5}$. In principle, color imagery has several benefits over monochrome imagery for surveillance, reconnaissance, and security applications. For instance, color may improve feature contrast, allowing better scene recognition and object detection. Color can be used to discriminate between different materials (e.g. in X-Ray baggage screening the sensor output at two energies for material classification). However, artificial coloring using colors that do not correspond in an intuitive way to those of a scene viewed under natural illumination may disrupt the recognition process. This may result in an observer performance that is even worse compared to that obtained with single band imagery alone ${ }^{6}$. Several different techniques have been proposed to display night-time imagery in natural daylight colors ${ }^{5,7-10}$, some of which have been implemented in realtime nightvision systems ${ }^{11-15}$. Most of these techniques are computationally expensive and do not achieve color constancy. Here we introduce a new method to apply natural daytime colors to multiband nightvision imagery. This method, for which a patent application is currently pending ${ }^{16}$, is simple and fast, can easily be deployed in realtime, and can display night-time imagery in natural daytime colors, that are stable under variations in scene content. The implementation in two prototype portable dual band realtime color night vision systems is described in an accompanying paper $^{17}$.

\section{COLOR MAPPING}

\section{A statistical coloring method}

Toet ${ }^{9}$ describes a statistical method for applying natural colors to a multiband night-time image. In this method certain statistical properties of a reference daytime image are transferred to the multiband night-time image. First, two or three bands of a multiband night-time image are mapped onto the RGB channels of a false color image. The resulting false color RGB night-vision image is then transformed into a perceptually de-correlated color space. In this color space the first order statistics (the mean and standard deviation of each channel) of a natural color image (target scene) are transferred to the multiband night-vision image (source scene). The inverse transformation back to RGB space yields a night-time image with a daytime color appearance. Figure 1 shows an example of this coloring scheme in which the two input night-time images (Figs. 1a and 1b) were taken with a NVG (night vision goggle) device in combination with respectively a low-pass and a high pass filter, splitting the NVG-sensitive range into a short (Fig. 1a) and a long wavelength part (Fig. 1b). Figure 1c shows the reference color image and Figure 1d shows the colorized night-time

* maarten.hogervorst@tno.nl; phone +31-346-356241; fax +31-346-353977 
image resulting from the application of the method described by Toet ${ }^{9}$. The advantage of this coloring scheme is the use of statistical image properties. The target image need not represent the same scene as the source image; it should merely be similar in composition and content.

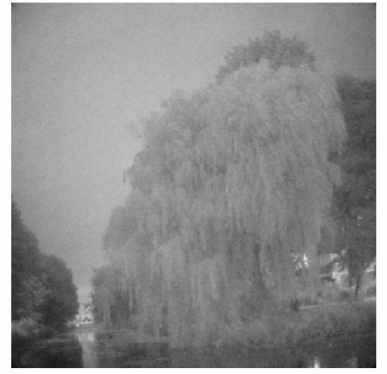

(a)

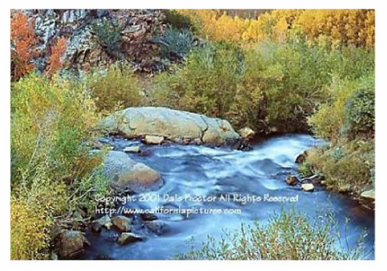

(c)

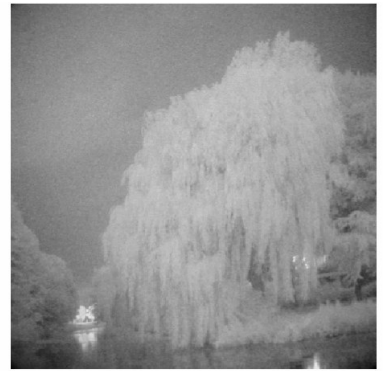

(b)

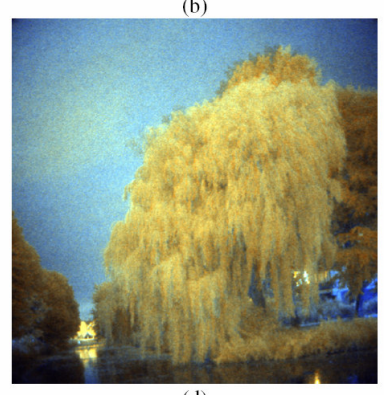

(d)

Figure 1. Example showing the coloring method described by Toet (2003) in which statistical properties of a reference image (Figure 1c) are transferred to a multiband sensor image. The multiband sensor consists of a NVG with filter transmitting wavelengths lower than $700 \mathrm{~nm}$ (Figure 1a) and an NVG sensor with filter transmitting wavelengths higher than $700 \mathrm{~nm}$ (Figure 1b). The color fused result is displayed in Figure 1d.

The use of color lookup tables

Figure 2a shows a (false color) representation of the multiband sensor image (Figure 1a and b) with the short wavelength part as the red and the long wavelength part as the green channel. Figure 2c shows all possible multi band sensor outputs (R-G-combinations) and Figure $2 \mathrm{~d}$ shows the corresponding color in the colorized image following the statistical coloring method of Toet ${ }^{9}$ for the example shown in Figure 1.

Instead of following the steps described by Toet $^{9}$ we propose to implement the coloring method using a color lookup transformation. In this method the false color RG image (Figure 2a) is translated into an indexed image using a color map containing all the colors of Figure 2c. When the color map is replaced by a color table containing all the colors in Figure $2 \mathrm{~d}$ the colorized image (Figure $2 \mathrm{~b}$ ) arises. Such a color lookup table transformation is very efficient and can be implemented in realtime. Moreover, the same color scheme can be applied to a different image. This assures that the color of objects no longer depend on the image content. This means for instance that the colors of objects do not change under panning and zooming operations. 

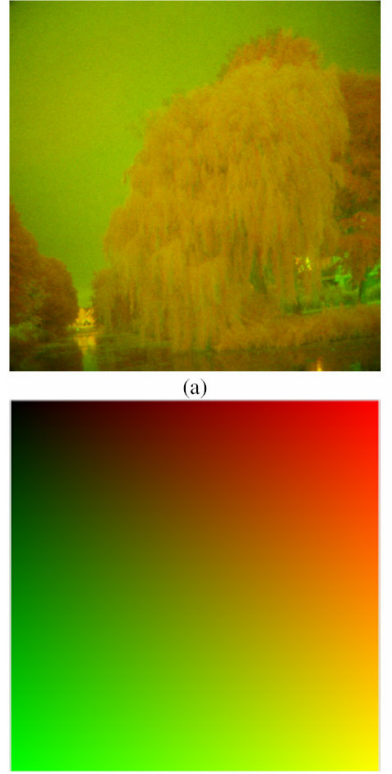

(c)

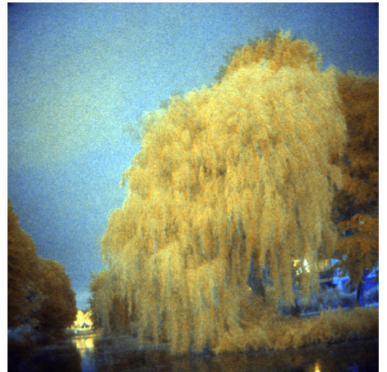

(b)

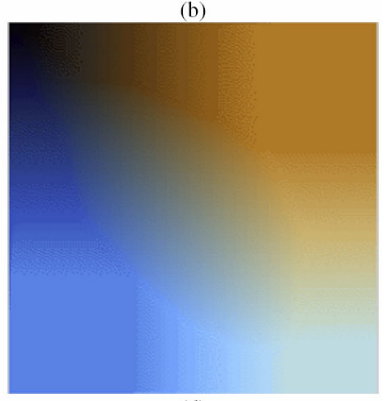

(d)

Figure 2. (a) False RG color representation of the sensor bands shown in Fig. 1a and 1b. (b) As Fig. 1d. (c) RG representation of all possible combinations of signal values in the two sensor bands. Upper left: both sensors give zero output; lower right: both sensors give maximal output. (d) Result of applying Toet's method to (c). This image represents all colors that can occur in the colorized sensor image (b).

\section{Coloring method based on samples}

We present a new coloring method that derives the color transformation from a set of samples for which both the multiband sensor values and the corresponding natural colors (RGB-value) is known ${ }^{16}$. The method results in colors that closely match the daytime reference colors. We will show how the color transformation can be derived from the combination of a multiband sensor image and a naturally colored image of the same scene, when the images are in correspondence. In that case, each pixel represents a sample for which both the multiband sensor values and the natural color are known.

Using the example shown in Figure 3 we will describe how the optimal color transformation can be derived (for details see ${ }^{16}$ ). Figure 3 a depicts the reference image, in this case a color photograph taken with a standard digital camera. We simulate a similar night-time sensor as depicted in Figure 1, i.e. a sensor consisting of two NVGs with filters transmitting wavelengths below and above $700 \mathrm{~nm}$. The first band contains the visible (short wavelength) part of the spectrum (see Figure 3b) while the other band contains the near infrared (NIR, long wavelength) part of the spectrum (Figure 3c). Figure $3 \mathrm{f}$ shows the result of applying day-light colors to the two-band night-time sensor image using our new color mapping technique.

The method is as follows. First, the multiband sensor image (is transformed to a false-color image by taking the individual bands (Figs. 3b and 3c) as input to the $\mathrm{R}$ and $\mathrm{G}$ channels (and $\mathrm{B}$ when the sensor contains three bands), referred to as the RG-image (Fig. 3e). Next, the resulting false color RG-image is converted to an indexed image using standard techniques. Each pixel in such an image contains a single index. The index refers to an RGB-value in a color lookup table (the number of entries can be chosen by the user). In the case of a sensor image consisting of two bands the color lookup table contains various combinations of R and B values (the B-values are zero when the sensor or sensor pair provides only two bands). For each index the corresponding natural color equivalent is derived by locating the pixels in the image with this index and finding the corresponding pixels in the (natural color) reference image (Fig. 3a). Next, we calculate the average color over this ensemble of pixels. One could simply take the average RGB-value. However, it's better to calculate the average in a perceptually de-correlated color space (e.g. $l \alpha \beta^{18}$ ) and convert the result to RGBspace. This assures that the computed average color reflects the perceptual average color. Averaging automatically takes the distribution of the pixels into account: colors that appear more frequently are attributed a greater weight. Finally, the 
RG-color table (left side of Fig. 3d) is replaced by the color table with natural colors (right side of Fig. 3d). This results in a two-band image with natural colors, in which the colors are optimized for this particular sample set (Fig. 3f). Note that the roof does not turn out orange in the colored night-time image. This is due to the fact that other materials that occupy a larger area of the image (the street) have a larger impact on the color scheme that is derived.

Instead of taking the average one could also take the median over the colors with the same index. Which of these (or other) solutions results in a better perceptual match depends on the image type and application. In principle, a cost function should be used that describes the similarity between the colorized image and the reference image (the average minimizes the squared error).

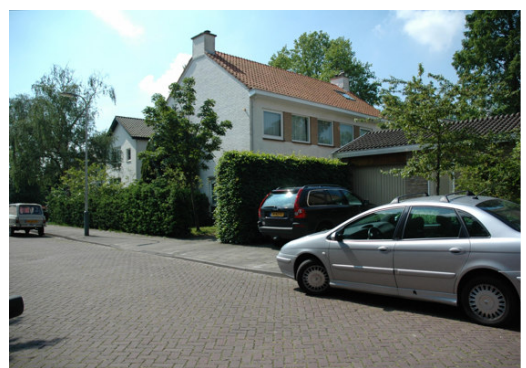

(a)

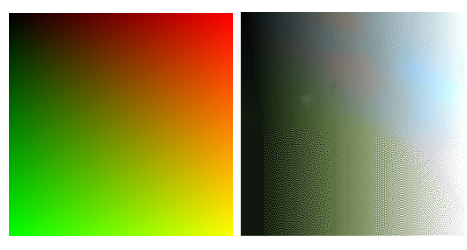

(d)

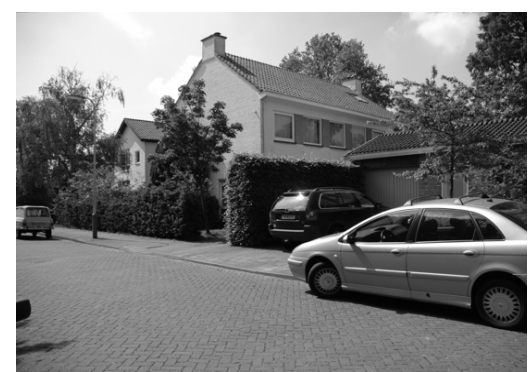

(b)

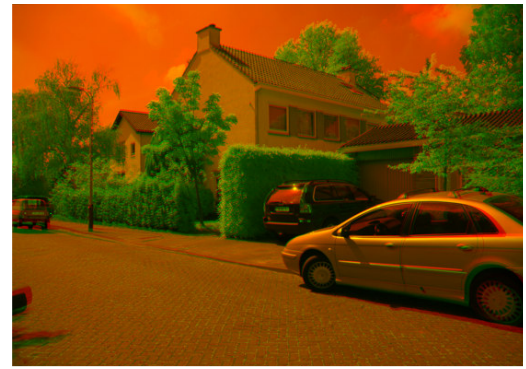

(e)

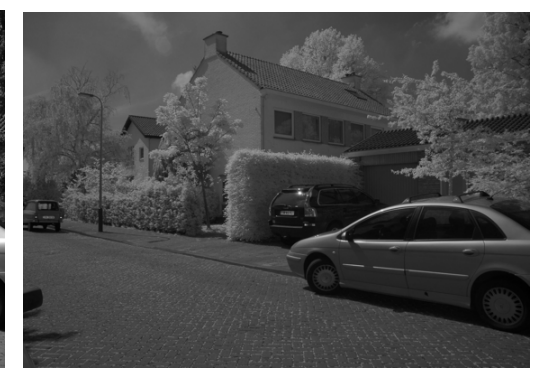

(c)

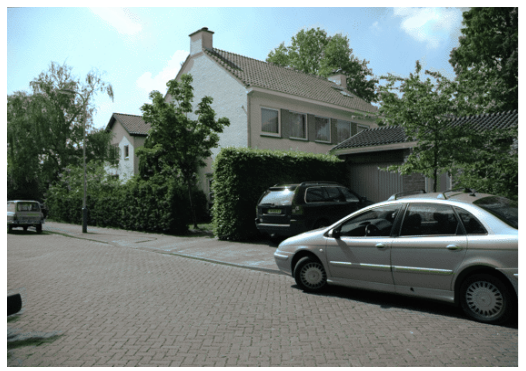

(f)

Figure 3. (a) Natural daylight color reference image. Visible (b) and near-infrared (c) band NVG images of the same scene.

(d) The color mapping derived from corresponding pixel pairs in (a-c). (e) Combined RG false color representation of

(b) and (c). (f) Result of the application of the mapping scheme in (d) to the false color image in (e).

The colors in the colorized two-band image closely match the colors in the reference image. This is due to the fact that the color mapping has been optimized for this particular reference image. However, the method only has practical value if it can be applied to other scenes as well. The first row (Figures 4a, 4b and 4c) shows the result of applying the same color transformation (i.e. using exactly the same color lookup tables) to different two-band night-time images (using the registration method described above). The second row (Figures $4 \mathrm{~d}, 4 \mathrm{e}$ and $4 \mathrm{f}$ ) shows the result of a color mapping that is optimized for the second scene ( $2^{\text {nd }}$ column). The third row (Figures $4 \mathrm{~g}$, $4 \mathrm{~h}, 4 \mathrm{i}$ ) shows the result of a color mapping that is optimized for the third scene $\left(3^{\text {rd }}\right.$ column). The fourth row shows the daytime reference images. Since the color schemes on the diagonal are optimized for the corresponding scene the images most closely resemble the daytime references. When a different color scheme is used certain objects appear with the wrong colors (e.g. in Figure $4 \mathrm{~g}$ the pavement is blue). Figure 5 shows the result of the different color schemes applied to the R-G image (see Figure $3 \mathrm{~d}$ ). High output in NIR and low output in the visible region is associated with a green color. This is due to the spectral reflectance of chlorophyll. 


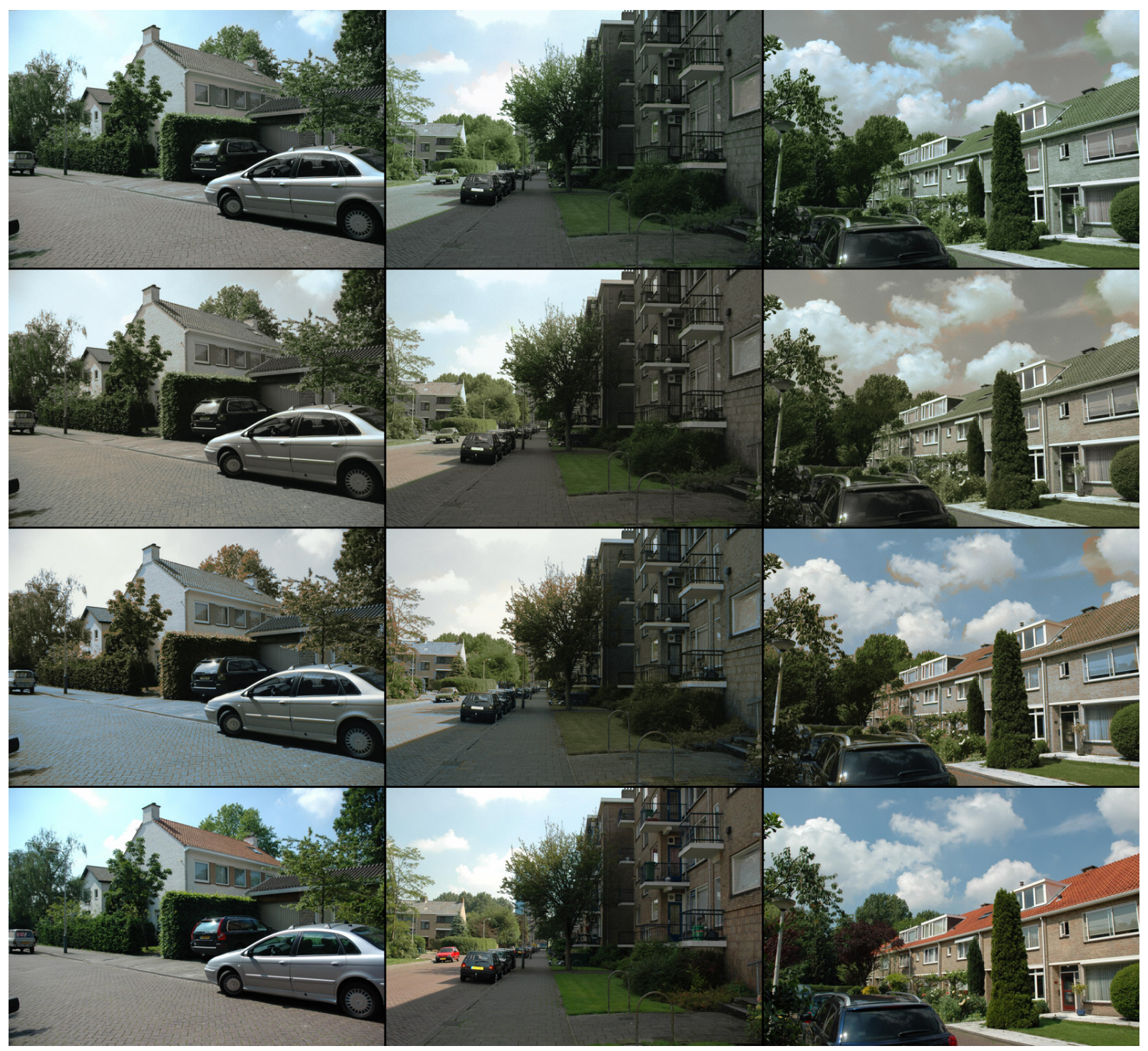

Figure 4. The result of the application of different color schemes to two-band night-time images (not shown) in which the color schemes are optimized for the first image (first row), second image (second row) and third image (third row). The fourth row shows the reference images.

a)

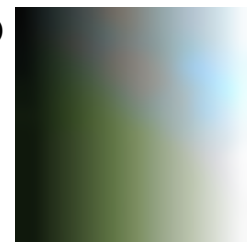

b)

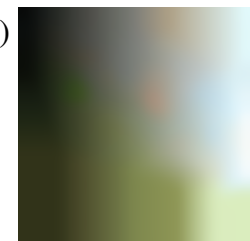

c)

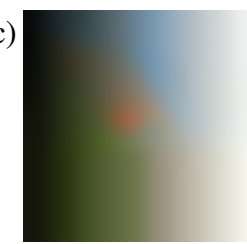

Figure 5. The result of the application of different color schemes to R-G image showing all sensor output combinations (see Figure $3 \mathrm{~d}$ ) in which the color schemes are optimized for the first image (a, first row), second image (b, second row) and third image (c, third row) of Figure 4.

One can also derive a color scheme that is suitable for most images by applying our method to a collage containing several images. Figure 6a shows the result of this procedure. The colors do not always precisely match the colors in the 
daytime references (Figure 6c; e.g. the hedge in the $2^{\text {nd }}$ image is reddish but is displayed as green in the colorized fusion image). Most important is that the color is characteristic for the material, enabling fast scene recognition and interpretation (diagnostic colors, i.e. grass should be green, but the exact shade of green is not important ${ }^{19-21}$ ). This becomes especially apparent when the (simulated) colorized twoband NVG image (Figure 6a) is compared to a standard NVG image (Figure 6d). The colorized image is much better in showing the layout of the scene and the different materials in the scene, thus facilitating situational awareness.

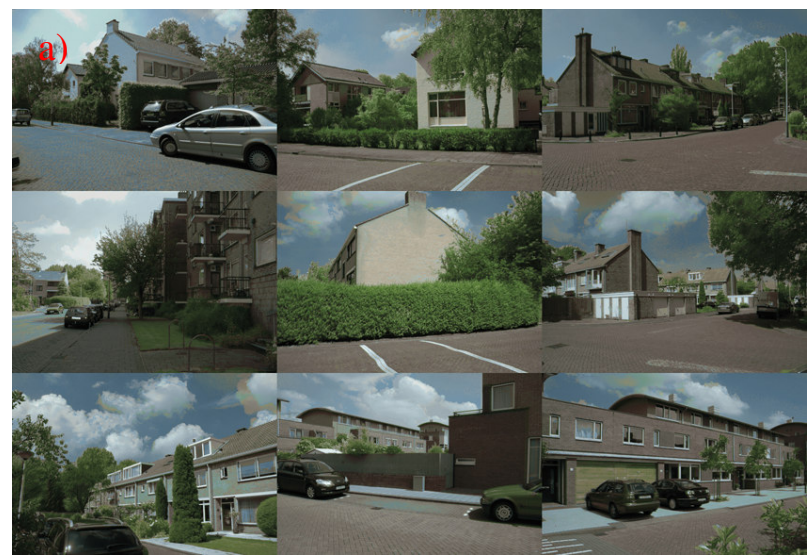

b)
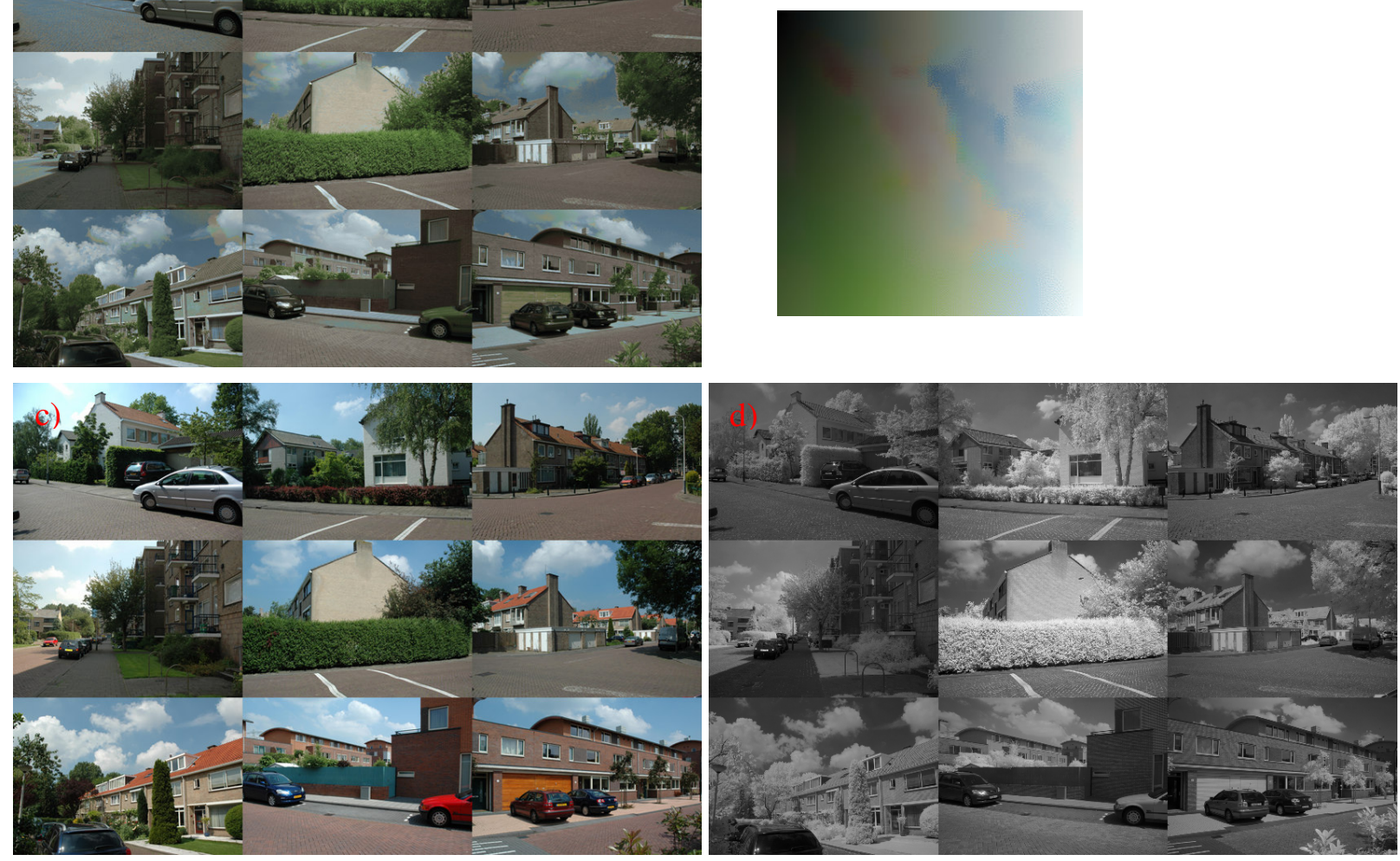

Figure 6. Result of a color scheme (represented in b) that is optimized to a range of images. a) shows the colorized multiband sensor image; c) shows the daytime color references. d) shows the NIR images for comparison between the two-band NVG sensor image and a standard NVG sensor image.

In the previous examples a two-band NVG system was simulated using images recorded during daytime. In a more critical test we used real NVG images recorded at night. Reference images showing the daytime colors were taken during daytime from the same viewpoint. Figure 7 shows two NVG images obtained with a filter transmitting wavelengths lower than $700 \mathrm{~nm}$ (Figure 7a) and higher than $700 \mathrm{~nm}$ (Figure 7b). Figure 7c shows the red-green false color representation of the two-band NVG image. Figure $7 \mathrm{~d}$ shows the daytime reference image corresponding to the multiband sensor image. Straightforward application of our method results in Figure 7e. The colors closely match the daytime colors (e.g. the sky is blue). However, the image looks noisy and certain objects appear in the wrong color (e.g. the bench and parts of the roof). This is due to the fact that the luminance in the colorized image does not increase continuously with increasing sensor output (the luminance in Figure 7c). This gives a "solarizing" effect which is undesirable. We therefore derived from this color map (inset in Figure 7e) another color map (inset in Figure 7f) in which the luminance increases linearly with the distance to the top-left corner. Figure $7 f$ shows the result of this new color mapping. The colors match the daytime colors. The sky is dark instead of light-blue. This corresponds to the intuition that of a dark sky at night and does not affect the situational awareness. 

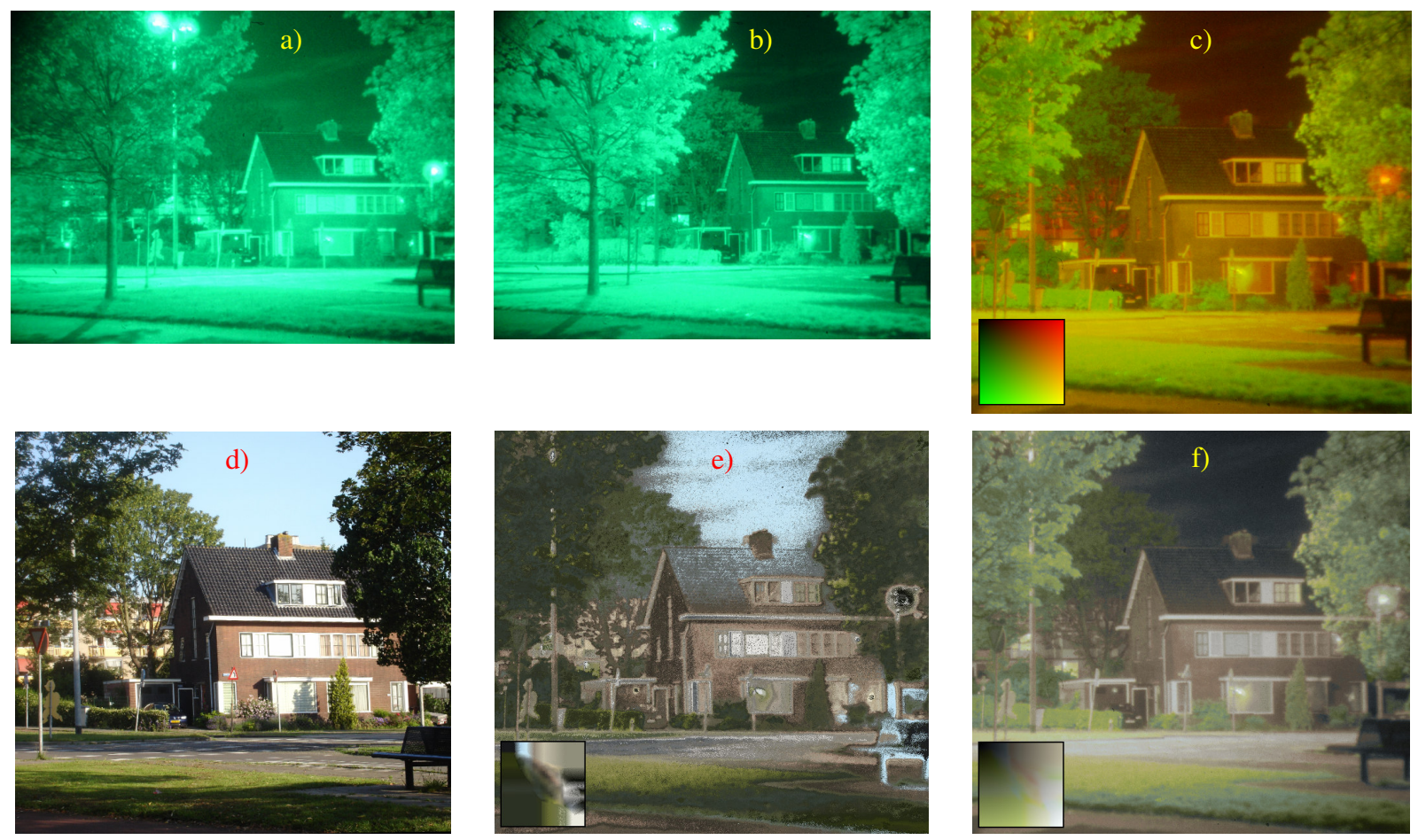

Figure 7. Example of application of the coloring method to a multiband nighttime image, consisting of two NVGs with filters dividing the sensitive range into a short wavelength part $(<700 \mathrm{~nm})$ and a long wavelength part $(>700 \mathrm{~nm})$ : a) short wavelength NVG image, b) long wavelength NVG image, c) red-green false color representation, d) daytime reference image, e) color fused image using the new coloring method straightforwardly, f) color scheme in which the luminance increases continuously with increasing sensor output.

Figure 8 shows the result of applying the same coloring scheme to different multiband sensor images. As in Figure 7, the colors do not always match the daytime colors (see Figure 9), but are representative for the different materials displayed in the scene. Thus, the colorized fused image facilitates interpretation of the scene (situational awareness). This is clear when comparing a normal NVG image (Figure 7b) with a two-band color fused image (Figure 7d).

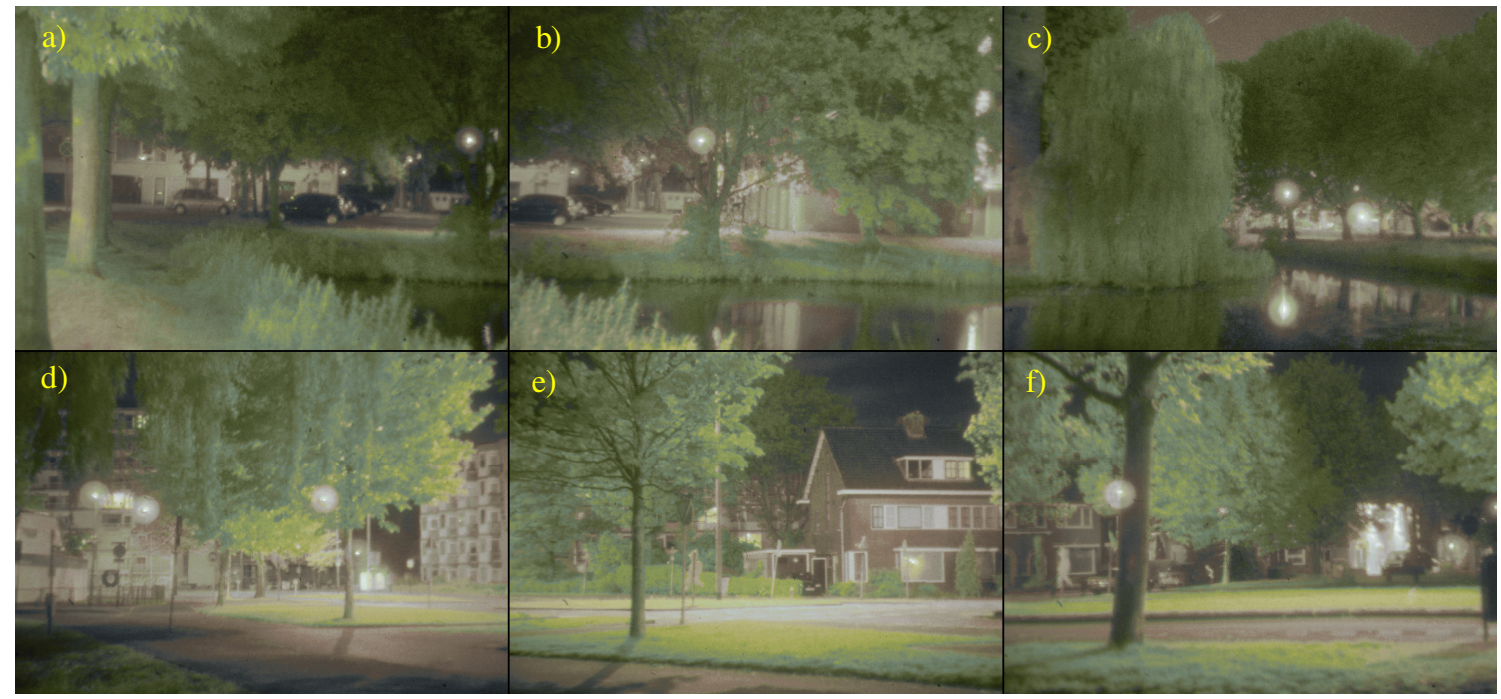

Figure 8. Result of the color scheme optimized for the image in Figure 7 applied to different other multiband sensor images. 


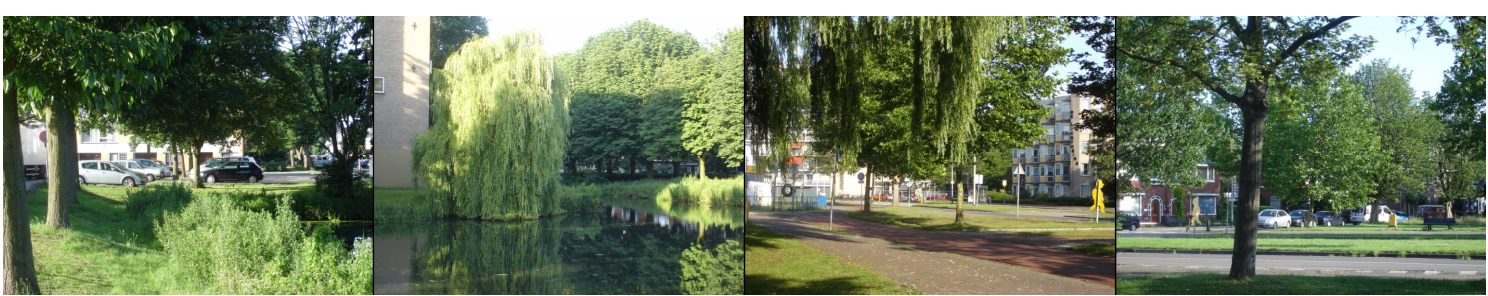

Figure 9. Daytime images corresponding to the color fused images displayed in Figure 8a, c, d, f.

\section{CONCLUSIONS}

We have presented a new method to apply natural daylight colors to multiband night-time images. The method derives an optimal color transformation from a set of samples taken from a daytime color reference image. The colors in the resulting colored multiband night-time image closely resemble the colors in the daytime color reference image. Moreover, when the same color scheme is applied to different multiband images there will be a close match between the colorized night-time images and their daylight color counterparts. Also, our method results in object colors that remain invariant under panning operations and are largely independent of the scene content.

The optimal color scheme for a certain type of environment should be derived in advance. During the night this color scheme can then be used to display night-time images in natural daytime colors. Different color schemes may be derived for different environments. The sample images used to derive the color scheme should reflect the natural statistics of the environment. The derivation of the color scheme may require some time. However, once the color scheme is derived the color lookup table technique allows for a realtime implementation that requires a minimal amount of processing time.

The similarity between a colorized night-time image and its natural daylight counterpart depends on the correlation between the sensor values and the daylight colors. A higher correlation results in more natural coloring. The natural colors are not well predicted when this correlation is low. This implies that a multiband sensor sensitive to wavelengths that are close to the visible range (such as NVG sensors with filters, see e.g. Figure 7 and 8) are most suitable for natural coloring. In contrast, the output of a sensor that is sensitive to the far infrared region of the spectrum will be less correlated with the daylight color. Therefore, a LWIR sensor is less suited for creating a fused image with daytime colors.

In an accompanying paper ${ }^{17}$ we describe the implementation of our color fusion method in two prototype portable dual band realtime night vision systems: the Gecko and the Viper system. The Gecko provides co-aligned visual and nearinfrared bands of two image intensifiers. The Viper provides co-aligned images from a digital image intensifier and an uncooled longwave infrared microbolometer. The co-aligned images from both systems are processed by a notebook computer. These prototypes demonstrate that the method can be applied in realtime. The Gecko shows that the natural colors make the image easier and faster to interpret. Also, these systems allow one to discriminate between different materials in the scene which are indistinguishable to a single band sensor. Whereas the intensified image of the Viper gives a good impression of the layout of the scene at night, the thermal image is highly useful for indicating potential (hot) targets. The color fused image of the Viper shows (hot) targets within their surroundings. The color scheme that is used in the Viper is optimized for discriminating different objects. In the near future we plan to build a demonstrator that incorporates two image intensifiers (with filters) with an infrared sensor. The output of the image intensifiers can be used to display the background in natural daytime colors, while the infrared sensor can be used to highlight potential (hot) targets.

\section{REFERENCES}

1. Li, G. and Wang, K., Applying daytime colors to nighttime imagery with an efficient color transfer method, In: J.G. Verly \& J.J. Guell (Ed.), Enhanced and Synthetic Vision 2007, pp. 65590L-1-65590L-12, The International Society for Optical Engineering, Bellingham, MA, 2007.

2. Shi, J., Jin, W., Wang, L. and Chen, H., Objective evaluation of color fusion of visual and IR imagery by measuring image contrast, In: H. Gong, Y. Cai \& J.-P. Chatard (Ed.), Infrared Components and Their Applications, pp. 594601, The International Society for Optical Engineering, Bellingham, MA, 2005. 
3. Shi, J.-S., Jin, W.-Q. and Wang, L.-X., Study on perceptual evaluation of fused image quality for color night vision, Journal of Infrared and Millimeter Waves, 24(3), pp. 236-240, 2005.

4. Tsagaris, V. and Anastasopoulos, D., Multispectral image fusion for improved RGB representation based on perceptual attributes, International Journal of Remote Sensing, 26(15), pp. 3241-3254, 2006.

5. Zheng, Y., Hansen, B.C., Haun, A.M. and Essock, E.A., Coloring night-vision imagery with statistical properties of natural colors by using image segmentation and histogram matching, In: R. Eschbach \& G.G. Marcu (Ed.), Color imaging X: processing, hardcopy and applications, pp. 107-117, The International Society for Optical Engineering, Bellingham, WA, 2005.

6. Sinai, M.J., McCarley, J.S. and Krebs, W.K., Scene recognition with infra-red, low-light, and sensor fused imagery, In: Proceedings of the IRIS Specialty Groups on Passive Sensors, pp. 1-9, IRIS, Monterey, CA, 1999.

7. Sun, S., Jing, Z., Li, Z. and Liu, G., Color fusion of SAR and FLIR images using a natural color transfer technique, Chinese Optics Letters, 3(4) ,pp. 202-204, 2005.

8. Tsagiris, V. and Anastassopoulos, V., Fusion of visible and infrared imagery for night color vision, Displays, 26(45),pp. 191-196, 2005.

9. Toet, A., Natural colour mapping for multiband nightvision imagery, Information Fusion, 4(3) ,pp. 155-166, 2003.

10. Wang, L., Jin, W., Gao, Z. and Liu, G., Color fusion schemes for low-light CCD and infrared images of different properties, In: L. Zhou, C.-S. Li \& Y. Suzuki (Ed.), Electronic Imaging and Multimedia Technology III, pp. 459466, The International Society for Optical Engineering, Bellingham, WA, 2002.

11. Wang, L., Zhao, Y., Jin, W., Shi, S. and Wang, S., Real-time color transfer system for low-light level visible and infrared images in YUV color space, In: I. Kadar (Ed.), Signal Processing, Sensor Fusion, and Target Recognition XVI, pp. 1-8, The International Society for Optical Engineering, Bellingham, WA, 2007.

12. Howard, J.G., Warren, P., Klien, R., Schuler, J., Satyshur, M., Scribner, D. and Kruer, M.R., Real-time color fusion of E/O sensors with PC-based COTS hardware, In: W.R. Watkins, D. Clement \& W.R. Reynolds (Ed.), Targets and Backgrounds VI: Characterization, Visualization, and the Detection Process, pp. 41-48, The International Society for Optical Engineering, Bellingham, WA, 2000.

13. Yue, Z. and Topiwala, P., Real-time EO/IR sensor fusion on a portable computer and head-mounted display, In: B.V. Dasarathy (Ed.), Multisensor, Multisource Information Fusion: Architectures, Algorithms, and Applications 2007, pp. 657106- The International Society for Optical Engineering, Bellingham, WA, 2007.

14. Fay, D.A., Waxman, A.M., Aguilar, M., Ireland, D.B., Racamato, J.P., Ross, W.D., Streilein, W. and Braun, M.I., Fusion of multi-sensor imagery for night vision: color visualization, target learning and search, In: Proceedings of the $3^{\text {rd }}$ International Conference on Information Fusion, pp. TuD3-3-TuD3-10, ONERA, Paris, France, 2000.

15. Waxman, A.M., et al., Solid-state color night vision: fusion of low-light visible and thermal infrared imagery, MIT Lincoln Laboratory Journal, 11 ,pp. 41-60, 1999.

16. Hogervorst,M.A., Toet,A., \& Kooi,F.L. (2006). TNO Defense Security and Safety. Method and system for converting at least one first-spectrum image into a second-spectrum image. 06076532 8-2202,

17. Toet, A. and Hogervorst, M.A., Portable real-time color night vision, In: B.V. Dasarathy (Ed.), Multisensor, Multisource Information Fusion: Architectures, Algorithms, and Applications 2008, pp. 1-1-1-, The International Society for Optical Engineering, Bellingham, WA, USA, 2008.

18. Ruderman, D.L., Cronin, T.W. and Chiao, C.-C., Statistics of cone responses to natural images: implications for visual coding, Journal of the Optical Society of America A, 15(8) ,pp. 2036-2045, 1998.

19. Goffaux, V., Jacques, C., Mouraux, A., Oliva, A., Schyns, P. and Rossion, B., Diagnostic colours contribute to the early stages of scene categorization: Behavioural and neurophysiological evidence, Visual Cognition, 12(6) ,pp. 878-892, 2005.

20. Oliva, A. and Schyns, P.G., Diagnostic colors mediate scene recognition, Cognitive Psychology, 41 ,pp. 176-210, 2000.

21. Rousselet, G.A., Joubert, O.R. and Fabre-Thorpe, M., How long to get the "gist" of real-world natural scenes?, Visual Cognition, 12(6) ,pp. 852-877, 2005. 